\title{
0 cuidado do idoso no contexto familiar: percepção da equipe de saúde da família*
}

Care of the elderly in the family context: the perception of the family health team

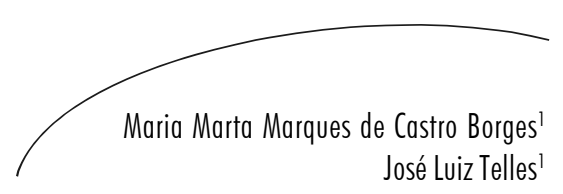

\section{Resumo}

Pesquisa qualitativa que utilizou estudo de caso e análise de conteúdo. Originalmente, teve como objetivo apreender o significado do cuidado domiciliário, na percepção dos profissionais de saúde da família, e suas interações com a família cuidadora de pessoas idosas dependentes. O objetivo deste artigo foi apresentar a experiência no cuidado domiciliário e as dificuldades a partir da percepção dos profissionais de saúde da família. Foi realizada na Região Metropolitana do Vale do Aço, Minas Gerais, nas cidades de Ipatinga, Timóteo, Coronel Fabriciano e Santana do Paraíso. Havia 54 equipes cadastradas na região e a amostra foi composta por 75 profissionais de saúde da família, auxiliares de enfermagem, enfermeiros, médicos e técnicos de enfermagem, que aceitaram participar da pesquisa, assinaram o TCLE e estavam atuando na equipe implantada há mais de um ano, após aprovação do gestor municipal. Os resultados apontaram que, na experiência da equipe, o cuidado domiciliar ao idoso dependente é realizado quando o mesmo se encontra num estágio de comprometimento da capacidade funcional mais avançado. Os profissionais apresentaram dificultadores como a demanda aumentada da USF, transporte insuficiente e equipe incompleta, e ainda se sentem despreparados para esse cuidado. Apresenta-se a necessidade de investimento dos gestores na educação continuada da equipe de saúde da família na área de geriatria e gerontologia, para que os profissionais deem conta de atender às necessidades dessa crescente população de idosos, que pode desenvolver fragilidade e comprometer sua autonomia e independência.

\section{Abstract}

Qualitative study that used case study and content analysis. Originally aimed to grasp the meaning of home care, in the perception of health of the family, and their interactions with family caregivers of elderly dependents. This paper aims to present the experience in home care and the difficulties from the viewpoint of family health

\footnotetext{
Universidade Católica de Brasília, Programa de Pós-graduação em Gerontologia. Brasília, DF, Brasil

* Texto-referência para a conferência proferida pelo Dr. José Luiz Telles na VII Jornada Brasileira de Enfermagem Geriátrica e Gerontológica, em 14/4/2010, na Universidade do Estado do Rio de Janeiro.
}

Palavras-chave:

Assistência Domiciliar. Idoso Fragilizado. Saúde da Família. 
professionals. It was conducted in the Metropolitan Region of Vale do Aço, Minas Gerais state, Brazil, in the cities of Ipatinga, Timóteo, Coronel Fabriciano and Santana do Paraíso. There were 54 teams registered in the region and the sample comprised 75 family health workers, nursing assistants, nurses, doctors and nurses, who agreed to participate in the study, signed the Informed Consent and were part of the staff for over one year after approval by the city manager. The results indicated that, according to the team's experience, home care for dependent elderly is performed when they are in a more advanced stage of impaired functional capacity. The subjects reported hindering the increased demand of USF, inadequate transport and incomplete team, and still feel unprepared for this care. There is the need for investment of managers in the continuing education of family health team in the area of geriatrics and gerontology for professionals to help them meet the needs of this growing elderly population, which may develop weakness and compromise their autonomy and independence.
Key Words: Home Nursing. Fragile Elderly. Family Health.

\section{INTRODUÇÃO}

Considerando a mudança do perfil etário da população, o Brasil apresentou, em 2007, o crescimento mais acentuado do grupo de pessoas com 75 anos ou mais de idade. As mulheres são maioria nesse grupo, numa razão de 79 homens para cada 100 mulheres ${ }^{1}$. Estima-se que 10 a $25 \%$ dos idosos acima dos 65 anos e $46 \%$ daqueles acima dos 85 anos, que vivem na comunidade, são pessoas frágeis ${ }^{2}$.

O termo fragilidade é utilizado em gerontologia e geriatria para indicar uma condição que possui maior prevalência nas pessoas mais idosas, do sexo feminino ${ }^{3,45}$, predispondo-as ao maior risco de eventos adversos, como a queda, e consequentemente, de hospitalização, declínio na sua funcionalidade com possibilidade de institucionalização e morte ${ }^{3,6}$.

Apesar disso, não há consenso sobre o conceito de fragilidade e não existe ainda validação para os critérios de avaliação da síndrome ${ }^{3,5,7}$. Mas a definição com que os profissionais da geriatria concordam, em um estudo brasileiro sobre as definições de fragilidade em idosos, apontou como uma "condição multifatorial de vulnerabilidade" possuindo o idoso diversas patologias e necessidade de uso de várias medicações e algum nível de dependência funcional e cognitiva. Consideraram que há possibilidade de prevenção, identificação e tratamento dos sintomas, mas que leva à maior morbidade e mortalidade ${ }^{7}$. Desta forma, a detecção precoce da fragilidade é determinante para intervenções precoces e, portanto, previne situações de maior debilidade física e funcional $^{8}$.

No grupo de idosos frágeis, a possibilidade de desenvolver dependência e incapacidade está aumentada, tornando-se a dependência uma consequência da fragilidade. Portanto, considerando que a fragilidade é uma condição progressiva, para uma parcela desses idosos a referência poderá ser tardia, quando se identifica a perda funcional ${ }^{6,9}$. Não há discordância, entre os pesquisadores, sobre o impacto negativo da fragilidade para as pessoas idosas e suas famílias, especialmente aos cuidadores e também para a sociedade como um todo ${ }^{5}$.

Devido a dificuldades econômicas e deficiências das políticas sociais ${ }^{10,11}$, nem sempre os muito idosos recebem a ajuda necessária da família, mesmo apresentando alguma incapacidade, e também não recebem apoio suficiente do Estado, sendo muitos deixados em situação de desamparo ${ }^{10}$.

Considerando que as pessoas idosas, em condições de fragilidade ou de maior vulnerabilidade, não comparecem com tanta frequência aos serviços de saúde, a modalidade de atenção domiciliária deve ser planejada, pela Estratégia de Saúde da Família, garantindo o vínculo com o sistema de saúde ${ }^{12}$. A responsabilidade da família se soma com o papel do Estado na promoção, proteção e recuperação 
da saúde nos três níveis de gestão do Sistema Único de Saúde (SUS).

Portanto, o modelo de cuidado domiciliário defendido na política do idoso demanda programas de orientação, informação e apoio de profissionais capacitados em saúde do idoso e é dependente do suporte informal familiar ${ }^{13}$. A assistência domiciliar com enfoque gerontológico deve ter em vista a promoção da autonomia e independência da pessoa idosa, estimulando-a para o autocuidado, em especial daqueles mais fragilizados e mesmo os sem perspectiva terapêutica de cura, atendendo a suas necessidades, contribuindo para melhorar sua qualidade de vida $^{12,13,14}$. A utilização deste espaço ocorre devido à necessidade de atender a demandas como a assistência individualizada, segurança e privacidade, redução das iatrogenias, maior controle da tomada de decisão, maior envolvimento da família e cliente com o planejamento e a execução do cuidado ${ }^{12,15}$.

Desta forma, os profissionais necessitam aprofundar no conhecimento de suas relações e estar habilitados para compreender o significado desse cuidado e compartilhar essas funções com o cuidador e familiares, dando-lhes assessoria para que possam dar conta das novas tarefas. Portanto, cabe aos profissionais de saúde apoiar a família, fortalecendo-a e orientando-a conforme suas necessidades ${ }^{16}$.

Apesar da existência de legislação específica ${ }^{13}$ abordando o cuidado domiciliar, as equipes $\mathrm{da}$ saúde da família encontram dificuldades e não estão preparadas para essa atividade no seu processo de trabalho ${ }^{14}$, como bem define a Política Nacional de Saúde da Pessoa Idosa. Há escassez de equipes multiprofissionais e interdisciplinares com conhecimento específico na área do envelhecimento e na saúde da pessoa idosa ${ }^{13}$. Tal situação acaba por produzir ações descoordenadas e mesmo intervenções inoportunas ou iatrogênicas. Assim, é necessária a qualificação profissional para aperfeiçoamento desse modelo de atenção $0^{9,15}$, redefinição das responsabilidades de cada esfera de governo, para a adequação de infraestrutura de atendimento, implementação de recursos tecnológicos e estabelecimento de regras de gestão, organização e financiamento do sistema ${ }^{17}$.

A questão de estudo que se apresenta é se os profissionais de saúde da família estão preparados para assistir a família cuidadora na dinâmica do processo de cuidar do idoso frágil, no contexto domiciliar, considerando as interações familiares.

Portanto, o objetivo deste artigo foi apresentar a experiência no cuidado domiciliário e as dificuldades, a partir da percepção dos profissionais de saúde da família e das suas interações com a família cuidadora de pessoas idosas dependentes.

\section{MATERIAL E MÉTODOS}

A pesquisa tem característica qualitativa e utilizou-se da modalidade de estudo de caso, pretendendo ampliar o conhecimento sobre a percepção dos profissionais de saúde sobre o cuidado domiciliar aos idosos dependentes.

A pesquisa foi realizada na Região Metropolitana do Vale do Aço, leste de Minas Gerais, considerando sua característica de conurbação, abrangendo as cidades de Ipatinga, Timóteo, Coronel Fabriciano e Santana do Paraíso.

O foco da pesquisa foi a atenção básica e, portanto, consideraram-se as equipes de saúde da família destes municípios para coleta de dados, que ocorreu de julho a agosto de 2009. Foram identificadas 54 ESFs no Cadastro Nacional de Estabelecimento de Saúde (CNES) e 50 equipes possuíam mais de um ano de implantação ${ }^{18}$.

A pesquisa foi realizada com os sujeitos trabalhadores da Estratégia de Saúde da Família da região metropolitana com mais de um ano de implantação, por entender que a partir deste período as equipes já tiveram a oportunidade de conhecer a população da área de abrangência e definir sua estratégia de trabalho.

Essa região possuía 454.053 habitantes e sua população idosa foi de 40.399 habitantes, 
representando $8,89 \%$ da população total, sendo 22.997 habitantes de 60 a 69 anos, 12.558 habitantes de 70 a 79 anos e 4.844 habitantes com 80 anos e mais ${ }^{19}$.

A população do estudo foi de 210 profissionais de Saúde da Família, incluindo os enfermeiros, médicos da família, auxiliares de enfermagem e/ ou técnicos de enfermagem. Os agentes comunitários de saúde não fizeram parte da população do estudo, pois não são os profissionais diretamente ligados ao cuidado dos idosos. Dessa maneira, o universo da pesquisa ficou constituído por 75 profissionais, sendo três auxiliares de enfermagem, 29 enfermeiros, 20 médicos e 23 técnicos de enfermagem, que agendaram horário para entrevista e aceitaram participar.

O projeto de pesquisa foi apresentado aos gestores municipais de saúde dos municípios da Região Metropolitana do Vale do Aço, com a assinatura do termo de autorização. Foram feitos os esclarecimentos sobre a pesquisa, a justificativa, objetivos e procedimentos a serem utilizados e os cuidados éticos e apresentado o parecer $\mathrm{n}^{\circ}$. CEP/ UCB O55/2009, do Comitê de Ética da Universidade Católica de Brasília.

Após contato telefônico com os gerentes das USF, foram agendados visita e encontro com os profissionais, conforme a disponibilidade de atendimento, sendo realizados os esclarecimentos. Após aceitarem participar, assinaram o Termo de Consentimento Livre eEsclarecido, com garantia de retirada da participação se o solicitassem, e de sigilo e anonimato. Os dados que se referiram ao perfil foram preenchidos pelo entrevistado na presença do pesquisador, e os formulários foram separados por categoria profissional sem a identificação dos sujeitos. As entrevistas foram gravadas, utilizando-se MP4 Imodo $2 \mathrm{~Gb}$, seguindo um roteiro de perguntas, e depois foram transcritas na íntegra.

Os cuidados éticos do estudo se fundamentaram nas definições da Resolução $n^{\circ}$ 196/96 do Conselho Nacional de Saúde ${ }^{20}$.

A análise dos dados primários foi realizada mediante a técnica de análise do conteúdo, e para a tabulação quantitativa foi utilizada a estatística descritiva simples.

Das categorias definidas no roteiro previamente preparado para atingir os objetivos a que se desejava chegar, identificaram-se as subcategorias a partir das unidades de registro que representaram o conteúdo das falas dos sujeitos da pesquisa e, após transcrição na íntegra, foram apresentadas nas unidades de contexto, sendo realizada limpeza dos vícios de linguagem para a utilização das falas no texto.

Do conteúdo das entrevistas, após leituras repetidas, foram extraídas as unidades de significação, apresentando-se a experiência dos sujeitos da pesquisa em relação ao cuidado domiciliar aos idosos dependentes e os fatores dificultadores desse cuidado.

\section{RESULTADOS}

Identificou-se inicialmente o perfil dos profissionais participantes da pesquisa. Os sujeitos foram os profissionais da saúde dos municípios da Região Metropolitana do Vale do Aço, trabalhadores das unidades de Saúde da Família, sendo que os auxiliares de enfermagem foram três (4\%), todos do município de Ipatinga; os enfermeiros foram 29 (38,7\%) e os médicos foram 20 (26,6\%). Os técnicos de enfermagem foram 23 (30,7\%).

Quanto ao tempo de formação, três (15\%) médicos tinham menos de um ano de formados; 38 (50,6\%) profissionais estavam na faixa de 1 a 4 anos de formação; 20 (26,6\%), de 5 a 10 anos, e $14(18,6 \%)$ tinham mais de 10 anos de formação.

Em relação ao tempo de atuação nas USF, 36 (48\%) profissionais estavam na faixa de 1 a 4 anos na mesma equipe, sendo $16(55 \%)$ enfermeiros, nove $(45 \%)$ médicos e $11(47,8 \%)$ técnicos de enfermagem. Destaca-se que oito (40\%) médicos e sete $(30,4 \%)$ técnicos de enfermagem estavam há menos de um ano na equipe.

Os conteúdos foram organizados e apresentados de acordo com as seguintes 
categorias: a) experiências vivenciadas no cuidado domiciliar; b) relações com os familiares; c) avaliação da estrutura e funcionalidade familiar; d) atividades específicas para idosos; e) fatores facilitadores do cuidado domiciliar; f) fatores dificultadores do cuidado domiciliar; e g) significado do cuidado domiciliar a esses idosos dependentes.

Serão apresentados neste artigo os resultados referentes a experiências no cuidado domiciliar ao idoso dependente e os fatores dificultadores desse cuidado.

Considerando a experiência no cuidado domiciliar, os profissionais expressaram três subcategorias, como: educação; dependência do idoso e organização do trabalho.

A ação educativa, abordada por 62 (82,6\%) dos entrevistados, foi organizada em unidades de significação, compreendendo: orientações aos idosos e familiares quanto ao uso de medicamentos e alimentação, quanto ao conforto e higiene, tratamento de feridas e prevenção de infecção e quanto à reabilitação, sendo esta abordagem com baixa ocorrência, com apenas dois (2,6\%). As abordagens inespecíficas foram excluídas da análise.

A dependência do idoso foi abordada por 42 (56\%) entrevistados e identificou-se a realização de visita domiciliar para idosos acamados portanto, com mobilidade física prejudicada. Este comprometimento foi apresentado como critério de inclusão no cuidado domiciliar. $\mathrm{Na}$ organização do trabalho, 37 (49,3\%) profissionais abordaram este aspecto, e o acompanhamento das patologias agudas e crônicas foi mais destacado.

Os fatores dificultadores para o cuidado domiciliar aos idosos dependentes foram apresentados por 72 (96\%) sujeitos da pesquisa, com maior variabilidade das respostas. Destacaram-se três subcategorias: aspectos relacionados às famílias; à organização do modelo assistencial e à gestão.

Considerando os aspectos relacionados às famílias, foram identificadas as relações familiares negativas e maus-tratos, com 37 (49,3\%); a baixa condição socioeconômica, com 23 (30,6\%) ocorrências; a falta de organização familiar em 17 (22,6\%); idosos que moram ou ficam sozinhos e o estresse dos cuidadores, ambos com 13 (17,3\%) sujeitos abordando a questão.

Nos aspectos relacionados à organização do modelo assistencial, destacou-se a demanda espontânea aumentada nas USFs, com 24 (32\%), e a falta de organização da referência e contrarreferência, que obteve 19 (25,3\%) ocorrências.

Quanto aos aspectos relacionados à gestão, a disponibilidade insuficiente de transporte foi abordada por $43(57,3 \%)$ profissionais; recursos humanos insuficientes, por 14 (18,6\%); e deficiência de capacitação da equipe, que foi identificada por $12(16 \%)$ sujeitos da pesquisa.

\section{DISCUSSÃO}

Os enfermeiros representaram o maior percentual da amostra, não havendo motivo específico para esta ocorrência; já em relação aos auxiliares de enfermagem, que representaram o menor percentual, considera-se que há hoje uma redução gradativa da categoria, pela possibilidade de acesso à qualificação no Vale do Aço. No entanto, os técnicos de enfermagem do município de Ipatinga são lotados nas equipes como auxiliares de enfermagem, conforme confirmado pelo Cadastro Nacional de Estabelecimentos de Saúde $(\mathrm{CNES})^{18}$.

Os profissionais do município de Ipatinga representaram o maior percentual da amostra para todas as categorias, sendo o fato explicado pelo maior número de equipes cadastradas.

Destaca-se o número de médicos e técnicos de enfermagem com menos de um ano de atuação na equipe, podendo-se inferir um período curto de experiência profissional.

Em relação a sua experiência nesse cuidado domiciliar, os profissionais se referiram às 
orientações para atender às necessidades básicas e de tratamento, independentemente de o usuário ser ou não idoso, conforme exemplificado:

"[...] a gente tenta enfocar mais em relação aos cuidados específicos aos acamados mesmo, o colchão, virar a posição, alimentação, as vezes é o próprio cuidado do ambiente, a gente percebe que o ambiente não está adequado, que não está ventilado e que não tá bigiênico”. (M 44)

Entende-se que o cuidado domiciliar implica compreender o contexto social, econômico e cultural dessas famílias, que envolve as modificações dos hábitos e seus significados perante a dinâmica familiar. Portanto, cuidar é mais que orientar. Cuidar é se comprometer com o outro e se responsabilizar ${ }^{21}$.

Destaca-se que os profissionais apontaram a eventualidade do cuidado domiciliar, conforme o discurso apresentado:

"[...] às vezes eu passo três, quatro quartas-feiras sem ver ninguém sabendo que existe." (M 39)

A eventualidade do cuidado sugere baixa densidade do vínculo, que é um componente essencial na ESF e, por conseguinte, compromete o princípio da integralidade. Estes dois conceitos fundamentam a organização do serviço e a renovação das práticas que valorizam o cuidado e que consideram o usuário, aqui representado pela pessoa do idoso, como sujeito a ser atendido e respeitado em suas demandas e necessidades ${ }^{22}$.

Destaca-se a relação feita pelos profissionais quanto ao grau de dependência do idoso e a necessidade do cuidado domiciliar. Este cuidado está direcionado aos idosos com mobilidade física comprometida, que não conseguem comparecer à unidade, estando comumente acamados, conforme apontado:

"[...] oprimeiro contato que a gente teve foi com os idosos acamados, a nossa preocupação era visitar esses idosos acamados, até com o pedido da família mesmo." (E 1)

O critério para inclusão do usuário na modalidade do cuidado domiciliar estabelece o desenvolvimento de algum grau de dependência, incluindo o comprometimento da mobilidade e permitindo a organização do cuidado para aqueles que necessitam ${ }^{2}$. No entanto, os usuários desta região metropolitana se apresentam numa condição de acamados quando recebem esse cuidado, encontrando-se num grau de dependência mais avançado. Pode-se inferir, portanto, que a assistência domiciliar tem ocorrido de forma tardia, pois quando se considera a perda da independência para a realização das intervenções, o grau de comprometimento desses idosos pode já estar adiantado ${ }^{3,6}$. É sabido que as pessoas idosas frágeis que mais se beneficiam das intervenções no cuidado domiciliar são as que ainda não são dependentes ou as que apresentam dependência inicial e estão em risco de progredir ${ }^{23}$. Quanto mais tardia a intervenção, maior será o risco de morte.

$\mathrm{Na}$ organização do trabalho da equipe, percebe-se o acompanhamento para controle das patologias crônico-degenerativas e para o tratamento de doenças agudas, ficando evidente no discurso apresentado:

\section{"[...] o idoso boje a gente vê ele mais no contexto da doença mesmo, quando ele vem e procura a gente com um motivo de doença." (M 30)}

Desta forma, identifica-se uma incoerência na percepção dos sujeitos em relação à proposta da Estratégia de Saúde da Família. Da forma como apresentam sua experiência, constata-se semelhança com o modelo assistencial das UBS tradicionais, centrado na doença e na intervenção médica ${ }^{24}$.

Trazendo o raciocínio da gerontologia e geriatria e as recomendações previstas na Política de Assistência ao Idoso, o modelo do cuidado está pautado na avaliação da funcionalidade, considerando a capacidade de autocuidado, a saúde física e mental, as condições socioeconômicas e ainda seu suporte social. A abordagem de consenso é que esta deverá ser ampla e que o foco não seja somente na doença, mas sim com uma visão integral da pessoa idosa, tendo a Estratégia de Saúde da Família ou a UBS como porta de entrada no SUS ${ }^{9,25}$. Em suma, os profissionais 
devem estabelecer como objetivo o desenvolvimento de ações que visem à manutenção da capacidade funcional do idoso, almejando plena habilidade física e mental, garantindo sua autonomia e independência ${ }^{13,25}$.

Para tanto, recomenda-se utilizar de instrumentos técnicos validados na captação do idoso e de sua família para o sistema de saúde, permitindo a identificação precoce dos seus problemas. Desta forma, o idoso será referenciado adequadamente com organização do atendimento e a priorização do cuidado para aqueles que mais necessitam ${ }^{2,26,27}$.

Ressalta-se que a Estratégia de Saúde da Família é um avanço na organização dos serviços de saúde e sua proposta busca a integração com a comunidade numa atuação interdisciplinar com compartilhamento dos saberes e otimização das ações da equipe ${ }^{22}$.

Os sujeitos da pesquisa destacaram as interações familiares negativas com referências aos maus-tratos com os idosos como um dos fatores dificultadores do cuidado domiciliar, conforme o discurso apresentado em relação aos aspectos da família:

"[...] a gente vê muita, muito abandono do idoso, muita negligência com relação ao idoso principalmente dentro da própria familia." (M 35)

Pessoas idosas, de todas as classes socioeconômicas, etnias e religiões, são vulneráveis aos maus-tratos, que podem ocorrer de várias formas, como: física, sexual, emocional e financeira, incluindo a negligência e o abandono ${ }^{28,29,30}$.

O cuidado com o idoso dependente, segundo o Estatuto do Idoso, é de responsabilidade da família ${ }^{31}$. E mesmo com a ocorrência de violência no ambiente domiciliar, este é o local de escolha deles para permanecer quando necessitam de cuidados $^{32}$. Portanto, espera-se que os profissionais da saúde da família se envolvam mais com as questões ligadas a violência, pois o pouco envolvimento se correlaciona ao fato de considerarem que é uma questão particular e portanto, da competência familiar ${ }^{28}$.
Conforme estabelecido no Estatuto do Idoso, compete ao profissional denunciar às autoridades os fatos que se configuram como negligência, omissões e maus-tratos, para que tomem providências para proteção dos idosos e punição dos infratores. ${ }^{28,31}$

A baixa condição socioeconômica, a falta de organização familiar, com a ausência do cuidador, e idosos morando ou permanecendo sozinhos a maior parte do tempo, ou cuidadores apresentando dificuldades de manutenção deste papel, também foram apontados como fatores dificultadores. Apresenta-se exemplo constatado pelos profissionais:

\section{"[...]. a dificuldade financeira, neste bairro é uma situação de imensa dificuldade, imensa."(M 41)}

Sabe-se que a organização dos serviços na modalidade de Saúde da Família tem ocorrido primeiramente nas regiões com condições socioeconômicas mais precárias ${ }^{24,33}$, e constatouse, na pesquisa, que os idosos frágeis sobrevivem com poucos recursos pessoais e sociais. Esta situação demonstra que o cuidado domiciliar formal se torna insuficiente para a qualidade de vida dessas pessoas.

No entanto, nem sempre o setor público está preparado para situações que surgem devido à falta de alternativas para o atendimento às necessidades desses idosos, como os profissionais apontaram:

"[...7 chama o Conselho do Idoso, e chama isso, chama aquilo, $e$ as coisas não vão acontecer, isto frustra a gente demais."(M 37)

Evidencia-se a necessidade de desenvolver novas formas de apoio formal aos cuidadores familiares, exercidas por instituições públicas ou privadas, e também para dar suporte aos profissionais da atenção básica, quando se deparam com os idosos que não possuem o suporte familiar ou não o recebem.

A situação apresentada pelos profissionais suscita a necessidade de gerar locais, equipamentos e técnicas que favoreçam o bem-estar dos idosos e dos que respondem por eles, garantindo 
qualidade na assistência ao idoso em situação de vulnerabilidade e, ainda, mantendo a saúde de quem cuida ${ }^{29,30}$.

Com relação à demanda aumentada, apontada como um dificultador, sabe-se que compromete a qualidade do cuidado domiciliar e interfere no planejamento do mesmo. No entanto, as estratégias de organização da oferta dos serviços prestados pelos profissionais daESF são semelhantes às da UBS tradicional, com agendamento utilizado como recurso operacional para ordenar as demandas apresentadas pela população e pautada em programas de controle de doenças crônicodegenerativas, como a hipertensão e diabetes ${ }^{24}$. Destacou-se a seguinte fala:

\section{"[...] dificulta mais ir as visitas é o fato da demanda} aqui no posto ser muito grande. Eu tenho uma demanda muito grande de pacientes que vem na segunda-feira de manhã fazero acolbimento e quando são muitos os pacientes que a gente não dá conta de atender acaba que ás vezes a gente marca pra tarde, então a gente deixa às vezes de fazer a visita pra poder atender a demanda, porque se eu saio pra visita eu vou atender dois, três acamados, quatro no máximo e se eu fico aqui, eu consigo atender quinze pessoas, então acaba que ás vezes isso acontece mesmo." (M 44)

A formação dos profissionais ainda apresenta um caráter centrado na doença e a Estratégia de Saúde da Família da região apresenta uma lógica de atendimento que prioriza produtividade, influenciando na organização do trabalho e interferindo na assistência ao idoso frágil.

Outro dificultador apresentado foi a falta de organização da referência e contrarreferência. Segundo o princípio da regionalização, os gestores devem organizar os serviços em três níveis de atenção à saúde: primária, com foco na atenção básica, preferencialmente no modelo da Saúde da Família; secundária, de média complexidade, e terciária, de alta complexidade, que assegure acesso universal e adequado no nível de atenção que corresponda ao problema apresentado pelo idoso ${ }^{34}$. Este princípio orienta a descentralização dos serviços de saúde e a pactuação entre os gestores.
Sabe-se que os municípios utilizam do sistema de agendamento para os especialistas, e o tempo de espera para esse atendimento ultrapassa com frequência ao tempo desejado ou recomendado para as necessidades do idoso, conforme apontado:

"[...] também de conseguir cirurgia, por exemplo, de fratura de colo de fêmur, a gente tem uma paciente aqui na nossa área que ficou é vários meses, pra conseguir uma cirurgia, por causa da questão de prótese."(M 38)

O grau de resolubilidade da equipe está relacionado à capacitação dos seus membros, aos recursos tecnológicos necessários e à capacidade de referenciar ao serviço em outro nível de assistência, permitindo uma abordagem integral, sendo influenciado pelo grau de organização política dos gestores municipais.

Considerando os aspectos de gestão, os profissionais apontaram as questões ligadas à insuficiência do transporte para possibilitar o cuidado domiciliar. Atualmente, o carro atende 10 equipes e então é disponibilizado um turno por semana para cada uma delas. A área extensa, com acessos difíceis e a grande demanda da unidade, aumenta a necessidade de meio de transporte, que nem sempre atende à necessidade para o cuidado domiciliar, como apontado por eles:
"[...] dificultador eu acho que mais com relação ao transporte mesmo, porque nem sempre a gente tem o transporte disponivel, a gente tem transporte uma vez.por semana só, mais é ... como eu disse, que a gente faz esse cuidado mais por demanda, de repente a gente tem que fazer mais vezes na semana e a gente não consegue o transporte." (E 15)

A situação retrata uma das dificuldades da gestão municipal em assegurar condições às ESFs, para que o cuidado domiciliar possa ser resolutivo e de qualidade, reduzindo os entraves para viabilizar o acesso dos profissionais aos domicílios dos idosos, quando necessário.

Foram destacados, ainda, os recursos humanos insuficientes, com ausência temporária de médicos e agentes comunitários de saúde: 
"[...] dificultador que en vejo, às vezes a própria estrutura de trabalho que a gente tem, seja ela física quanto recursos bumanos, hoje você tem recursos bumanos amanhã não tem mais, então acaba gerando uma certa instabilidade em matéria de dificuldade do próprio conbecimento das famílias que é o que preconizam o PSF.”(E 19)

A proposta do modelo assistencial inclui equipe mínima, com necessidade de delimitação da área de abrangência e definição do número de pessoas nesta área, sendo de 3 mil a 4.500 pessoas ou 1.000 famílias. Desta forma, a população será assistida com qualidade e resolubilidade ${ }^{35}$.

A demanda aumentada e a equipe incompleta representam, mais uma vez, dificuldade na organização do processo de trabalho, incluindo o cuidado domiciliar. Este cuidado pode ser considerado um ato que requer o apoio e suporte da gestão pública dos serviços de saúde, para que os profissionais possam dar conta dessa assistência de maneira resolutiva e integral.

A questão da falta de capacitação profissional em gerontologia e geriatria foi abordada como fator dificultador do cuidado domiciliar, e foi apontada pelos sujeitos:

"[... também eu acho que capacitação éessencial, da equipe toda, acho que é essencial, para se ter noção e consciência de qualé sua atribuição, é importante."(E 21)

"[...] questão de formação profissional, é de capacitação mesmo, voltado pro idoso, principalmente na questão de medicação, a medicação do idoso ela é diferenciada, ela não é igual a medicação do adulto comum.”(M 38)

A necessidade de mudança do modelo centrado na doença e no profissional médico, para o modelo que estabelece a capacidade funcional e a ação multiprofissional como foco da assistência ao idoso, e ainda estabelecer o seu domicílio como local de abordagem, se depara com a falta de conhecimento dos profissionais de saúde em relação ao envelhecimento, por deficiência nas diretrizes curriculares no seu período de formação. O modelo até então vigente coloca em segundo plano os aspectos sociais, econômicos e subjetivos, que são determinantes do processo saúde doença $a^{25}$.

Torna-se então um desafio, considerando que o envelhecimento populacional é uma realidade, e as mudanças que possam ser introduzidas na formação dos profissionais não beneficiarão os idosos de agora. Portanto, para dar conta de atender a essa demanda com qualidade, a educação permanente na área do envelhecimento, que habilite os profissionais para lidar com as especificidades da população idosa, é uma responsabilidade dos gestores e está definida no Pacto pela Saúde ${ }^{36}$.

A educação permanente pode ocorrer de diversas maneiras, uma das quais é a abordagem de problemas, resgatando os conhecimentos dos profissionais, adquiridos na graduação. Nesta modalidade, a presença de um facilitador para educação permanente contribui para o desenvolvimento do grupo. Há ainda a educação à distância (EAD), que pode atender a um grande número de equipes, prestando-se à atualização dos profissionais, amplamente divulgada nos dias atuais. Ainda, pode-se utilizar da autoinstrução, com formação de grupos para compartilhar as experiências e a facilidade de acesso aos conteúdos institucionais via web ${ }^{37}$.

É comum os idosos serem atendidos por profissionais inexperientes e pouco qualificados para este cuidado ${ }^{38}$, conforme identificado na pesquisa.

Portanto para intervir nesta realidade, as recomendações são que os profissionais devam conhecer a diversidade do processo de envelhecimento, conhecer os avanços tecnológicos da área; reconhecer a importância $\mathrm{da}$ ação interdisciplinar; contribuir com programas de educação para o envelhecimento; assumir os desafios na promoção da saúde, do bem-estar e da qualidade de vida do idoso e promover ações educativas para mudanças de atitudes sobre a velhice e o envelhecimento, diretrizes que são enunciadas na Política Nacional do Idoso ${ }^{38}$. 


\section{CONCLUSÃO}

Os aspectos apresentados neste artigo fazem parte da dissertação do Mestrado em Gerontologia da Universidade Católica de Brasília. Pretendeuse, portanto, apresentar a experiência no cuidado domiciliário de pessoas idosas dependentes e as dificuldades vivenciadas para esse cuidado, a partir da percepção dos profissionais de Saúde da Família.

Considera-se oportuno lembrar que, no período da coleta de dados, os municípios de Ipatinga e Timóteo, que apresentaram o maior número de equipes de Saúde da Família, se encontravam em situação de instabilidade política, devido a processos na Justiça Eleitoral, que interferiram negativamente no gerenciamento dos diversos setores da Prefeitura Municipal, incluindo a Saúde.

Constatou-se que a atuação dos profissionais prioriza o modelo da UBS tradicional, com atendimento voltado para ações de caráter curativista, segmentado e com o foco nas patologias agudas ou crônicas apresentadas pelos idosos. O cuidado domiciliar é realizado quando

\section{REFERÊNCIAS}

1. Instituto Brasileiro de Geografia e Estatística. Estudos e Pesquisas, Informação Demográfica e Socioeconômica. Síntese de Indicadores Sociais. Uma análise das condições de vida da População Brasileira. Rio de Janeiro; 2008. Disponível em: URL: http://www.ibge.gov.br/ home/estatistica/populacao/condicaodevida/ indicadoresminimos/sinteseindicsociais2008/ indic_sociais2008.pdf.

2. Brasil. Ministério da Saúde. Secretaria de Atenção a Saúde. Departamento de Atenção Básica. Envelhecimento e Saúde da Pessoa Idosa. Caderno da Atenção Básica no. 19. Brasília. DF: 2007. 192p. Disponível em: URL: http:// bvsms.saude.gov.br/bvs/publicacoes/ abcad19.pdf.

3. Rockwood K, et al. A global clinical measure of fitness and frailty in elderly people CMAJ 2005; 173(5):M489-95. Disponível em: URL: http:// www.cmaj.ca. os idosos já se encontram com dependência avançada, por se apresentarem na condição de acamados. O termo fragilidade não é abordado por eles no contexto da pesquisa, demonstrando desconhecimento sobre este conceito.

Sentem-se despreparados para o cuidado domiciliar, mas há uma procura restrita em atualização e preparo técnico para a execução deste cuidado e na organização do trabalho da equipe para tal atividade. Assim, há necessidade de investimento na organização do apoio às equipes, garantindo sua mobilidade e acessibilidade para o cuidado domiciliar, bem como disponibilizando a qualificação permanente dos profissionais, instrumentalizando-os para o exercício de suas competências técnicas no atendimento à população idosa.

Reverter esta situação torna-se urgente, considerando a necessidade dos cuidadores e familiares em relação ao apoio formal no cuidado domiciliar e o aumento significativo da parcela dos muito idosos que poderão necessitar desta modalidade do cuidado, pois apresentam o risco mais elevado do desenvolvimento da síndrome clínica de fragilidade.

4. Fried LP, et al. Frailty in Older Adults: Evidence for a phenotype. J Gerontol 2001; 56A(3): M146-156. Disponível em: URL: http:// biomedgerontology.oxfordjournals.org/content/ 56/3/M146.full.

5. Karunananthan S, et al. A multidisciplinary systematic literature review on frailty: overview of the methodology used by the canadian initiative on frailty and aging. BMC 2009; 9(68). Disponível em: URL: http://www.biomedcentral.com/1471-2288/ $9 / 68$.

6. Jones DM, Song X, Rockwood K. Operationalizing a frailty index from a standardized comprehensive geriatric assessment. JAGS 2004 nov; 52(11):1929-1933. Disponível em: URL: http://www.sld.cu/ galerias/pdf/sitios/gericuba/ indice_de_fragilidad.pdf. 
7. Teixeira INDO. Percepções de profissionais de saúde sobre duas definições de fragilidade no idoso. Ciên. Saúde Coletiva 2008; 13(4):1181-88. Disponível em: URL: http://www.scielosp.org/ scielo.php?script $=$ sci anttext\&pid $=S 141381232008000400014$.

8. Veras RP, et al. Promovendo a Saúde e Prevenindo a Dependência: identificando indicadores de fragilidade em idosos independentes. Rev. Bras.Geriatr. Gerontol 2007; 10(3):1-22. Rio de Janeiro. Disponível em: URL: http://www.unati.uerj.br/tse/ scielo.php?script $=$ sci_arttext\&pid $=$ $\mathrm{s} 180998232007000300008 \& \operatorname{lng}=\mathrm{pt} \& \mathrm{nrm}=$ iso.

9. Lourenço A. Síndrome de Fragilidade no Idoso: marcadores clínicos e biológicos Rev Hosp Universt Pedro Ernesto, UERJ 20087 jan-jun; Disponível em: URL: http:// www.lampada.uerj.b/revistahupe/images/ ediçoes/an7_jan2008/artigo_2.pdf

10. Ferreira JVC. Os muito idosos no Município de São Paulo. 2006. [Dissertação Mestrado] Programa de pós graduação em saúde púbica. USP Faculdade de saúde pública; 2006. Disponível em: URL: http://www.teses.usp.br/ teses/disponiveis/6/6132/tde01022007174148/

11. Duarte YAO, Lebrão ML, Lima FD. Contribuição dos arranjos domiciliares para o suprimento de demandas assistenciais dos idosos com comprometimento funcional em São Paulo, Brasil. Rev Panam Salud Publica 2005; 17 (5/6): 370-378. Disponível em: URL: http:// www.scielosp.org/ scielo.php?script $=$ sci_arttext\&pid $=$ $\mathrm{S} 102049892005000500009 \& \operatorname{lng}=\mathrm{pt} \& \mathrm{Zn} r \mathrm{~m}=$ iso\&tlng $=\mathrm{pt}$.

12. Duarte YAO, Diogo MJD. Atendimento domiciliário: um enfoque gerontológico. São Paulo: Atheneu; 2005. 630 p.

13. Portaria GM no 2.528 de 19 de outubro de 2006. Política Nacional de Saúde da Pessoa Idosa. Brasília 2006. Disponível em: URL: http:// portal.saude.gov.br/portal/arquivos/pdf/ $2528 \% 20$ aprova $\% 20 a \% 20$ politica $\% 20$ nacional $\%$ 20de\%20saude\%20da\%20pessoa\%20idosa.pdf.

14. Floriani CA, Schramm FR. Atendimento Domiciliar ao idoso: problema ou solução? Cad. Saúde Pública 2004 jul-ago; 20(4). Disponível em: URL: http://www.scielosp.org/ pdf/csp/v20n4/13.pdf.

15. Moreira MD, Caldas CP. A importância do cuidador no contexto da saúde do idoso. Esc. Anna Nery R Enfern. 2007; 11(3):520-525. Disponível em: URL: http://www.eean.ufrj.br/ revista_enf/2007_vol11/ 2007 _vol11n03SETTEMBRO.pdf.
16. Elsen I, Marcon SS, Silva MRS. O viver em família e a sua interface com a saúde e a doença. Maringá: Eduem, 2004. 398 p.

17. Pinheiro R, Mattos RA, et al. Os sentidos da integralidade na atenção e no cuidado à saúde. 6. ed. Rio de Janeiro: IMS/UERJ - CEPESC ABRASCO, 2006. 180p. Disponível em: URL: http://www.lappis.org.br/cgi/cgilua.exe/sys/ start.htm?sid=25\&infoid = 180\&tpl = viewparticipantes.

18. Datasus. Cadastro Nacional de Estabelecimentos de Saúde. 2010. Disponível em: URL: http://tabnet.datasus.gov.br/cgi/ tabcgi.exe?cnes/cnv/equipemg.def.

19. Instituto Brasileiro de Geografia e Estatística. Cidades. Estimativa populacional dos municípios de Minas Gerais. 2009. Disponível em: URL: http://www.ibge.gov.br/cidadesat/ topwindow.htm?1.

20. Resolução n .196 de 10 de outubro de 1996. Aprova as diretrizes e normas regulamentadoras de pesquisas envolvendo seres humanos. Brasília. DF, 1996. Disponível em: URL: http:// conselho.saude.gov.br/resolucoes/1996/ Reso196.doc.

21. Boff L. Saber cuidar: ética do humanocompaixão pela terra. Petrópolis: Vozes, 5. ed. 2001.199p.

22. Gomes MCPA, Pinheiro R. Acolhimento e vinculo: práticas de integralidade na gestão do cuidado em saúde em grandes centros urbanos. Interface - comunic, Saúde, Educ 2005 mar-ago; 9 (17):287. Disponível em: URL: http:// www.scielo.br/pdf/\%0D/icse/v9n17/ v9n17a06.pdf.

23. Ferrucci L, et al. Designing randomized, controlled trials aimed at preventing or delaying functional decline and disability in frail, older persons: a consensus report. J Am Geriatr Soc 2004 apr; 52(4):625-634. Disponível em: URL: http://www.ncbi.nlm.nih.gov/pubmed/15066083.

24. Pinheiro R. As práticas do cotidiano na relação oferta e demanda dos serviços de Saúde: um campo de estudo e construção da integralidade. Rio de Janeiro. IMS/UERJ - CEPESC ABRASCO, 2006. Disponível em: URL: http:// www.lappis.org.br/cgi/cgiluaexe/sys/ start.htm?sid $=25 \&$ infoid $=180 \& \mathrm{tpl}=$ view_participantes.

25. Telles JL. Perspectivas e desafios para o planejamento das ações do Pacto pela Vida, Saúde da população idosa 2006/2007: contribuições para o debate. 2006. Disponível em: URL: http://www.sbggpr.org.br/artigos/ acoes_estrategicas_para implementar_politica_2007\%5B1\%5D.pdf. 
26. Costa EF, Monego ET. Avaliação Geriátrica Ampla (AGA). Revista da UFG 2003 dez; 5(2). Disponível em: URL: http://www.proec.ufg.br/ revista_ufg/idoso/aga.html.

27. Veras R. Em busca de uma assistência adequada à saúde do idoso: revisão da literatura e aplicação de um instrumento de detecção precoce e de previsibilidade de agravos. Cad. Saúde Pública 2003 maio-jun; 19(3):705-715. Rio de Janeiro. Disponível em: URL: http:// www.scielo.br/ scielo.php?script $=$ sci_arttext\&pid $=$ S141381232004000400024\&lng $=p t$

28. Minayo MCS. Violência contra idosos: relevância para um velho problema. Cad. Saúde Pública 2003 maio-jun; 19 (3):783-791. Rio de Janeiro. Disponível em: URL: http:// www.scielo.br/pdf/csp/v19n3/15881.pdf.

29. Faleiros VP. Violência contra a pessoa idosa ocorrências, vítimas e agressores. Brasília: Universa. 2007.394 p.

30. Sommerhalder C. Significados associados à tarefa de cuidar de idosos de alta dependência no contexto familiar. [Dissertação mestrado]. Universidade Estadual de Campinas. Faculdade de Educação. 2001. Disponível em: URL: http:// libdigi.unicamp.br/document/ ?code $=$ vtls000218732.

31. Lei ${ }^{\circ} 10.741$ de $1^{\circ}$ de outubro de 2003. dispõe sobre o estatuto do idoso e dá outras providências. Brasília: senado federal. 2003.

Disponível em: URL: http:// www6.senado.gov.br/legislacao/ listapublicacoes.action?id $=237486$

32. Sesc. Idosos no Brasil Vivencias, desafios e expectativas na terceira idade. São Paulo: FPA, 2007.
33. Piccini RX, et al. Necessidades de saúde comuns aos idosos: efetividade na oferta e utilização em atenção à saúde. Ciências e Saúde Coletiva 2006; 11(3):657-667. Disponível em: URL: http://www.scielo.br/pdf/csc/v11n3/ 30981.pdf

34. Brasil. Ministério da Saúde. Secretaria de Atenção à Saúde. Departamento de Regulação, Avaliação e Controle de Sistemas. Diretrizes para a programação pactuada e integrada da assistência à saúde. Brasília: Ministério da Saúde, 2006. 148 p. Disponível em: URL: http:// bvsms.saude.gov.br/bvs/publicacoes/ DiretrizesProgPactuadaIntegAssistSaude.pdf.

35. Brasil. Ministério da Saúde. Atenção Básica e a Saúde da Família. 2010 [on line] Disponível em: URL: http://dab.saude.gov.br/ atençaobasica.php\#diretriz.

36. Portaria 399 de 28 de fevereiro de 2006. Divulga o Pacto pela Saúde. Consolidação do SUS e aprova as Diretrizes Operacionais do Referido Pacto. Brasília. DF, 2006. Disponível em: URL: http://www.brasilsus.com.br/legislacoes/gm/ 992-399?q=

37. Costa Neto MM (org.). Educação Permanente. Brasília. 2000. Disponível em: URL: http:// bvsms.saude.gov.br/bvs/publicacoes/ cad03_educacao.pdf.

38. Neri AL, Jorge MD. Atitudes e conhecimentos em relação à velhice em estudantes de graduação em educação e em saúde: subsídios ao planejamento curricular. Estud. Psicol. Campinas 2006 abr-jun; 23 (2):127-137. Disponível em: URL: http://scielo.bvspsi.org.br/ scielo.php?script $=$ sci_arttext\&pid $=$ S0103166X2006000200003. 